Ann. Zootech., I975, $24(4), 645^{-6} 50$.

\title{
FERTILITÉ ET PROLIFICITÉ DE TRUIES INSÉMINÉES AVEC DU SPERME CONGELÉ
}

\author{
M. PAQUIGNON et M. COUROT* \\ avec la collaboration technique de J. Gautier et A. Locaterli \\ I. T. P., 149, wue de Bercy, \\ 75579 Paris Cedex 12 \\ * Station de Recherches sur la Physiologie de la Reproduction, \\ Centre de Recherches de Tours, I. N. R. A., \\ Nouzilly, 37380 Monnaie
}

RÉSUMÉ

Trois techniques de préparation de la semence avant la congélation sont étudiées afin de tester le pouvoir fécondant des spermatozoïdes. Les éjaculats entiers de 9 verrats sont partagés en 3 fractions $(\mathrm{A}, \mathrm{B}, \mathrm{C})$ et centrifugés à $800 \mathrm{~g}$ pendant $15 \mathrm{mn}$ à $30^{\circ} \mathrm{C}$. Le culot est dilué une première fois avec un dilueur jaune d'œuf glucose non glycérolé puis la semence est mise à refroidir selon deux vitesses : 1 tot $\mathrm{A}: 30^{\circ} \mathrm{C}$ à $15^{\circ} \mathrm{C}$ en I heure, $15^{\circ} \mathrm{C}$ à $5^{\circ} \mathrm{C}$ en I heure. Lots $\mathrm{B}$ et $\mathrm{C}$ : $30^{\circ} \mathrm{C}$ à $I 5^{\circ} \mathrm{C}$ en I heure, maintien à $15^{\circ} \mathrm{C}$ pendant 4 heures et abaissement à $5^{\circ} \mathrm{C}$ en $\mathrm{I}$ heure. La semence est rediluée avec le même dilueur glycérolé à $\mathrm{I} 5^{\circ} \mathrm{C}$ après $\mathrm{r}$ heure de refroidissement pour les lots $A$ et $B$ et 5 heures de refroidissement pour le lot $C$. La semence est congelée en pastilles de $\mathrm{o}, \mathrm{I} \mathrm{ml}$. Les doses d'inséminations sont décongelées dans le dilueur INRA-ITP à $50^{\circ} \mathrm{C}$ (I/5 en volume). 63 truies nullipares sont inséminées 34 heures après le début présumé des chaleurs avec 3 à $4 \cdot \mathrm{IO}^{9}$ spermatozoïdes mobiles.

Le maintien de la semence pendant 4 heures à $I 5^{\circ} \mathrm{C}$ améliore d'une façon significative $(\mathrm{P} \leqslant 0,05)$ le taux de gestation $(63,6 \mathrm{p}$. 100 et 66,0 p. Ioo pour les lots $\mathrm{B}$ et $\mathrm{C}$ contre 50 p. Ioo pour le lot A).

L'augmentation de 4 heures de la durée de contact des spermatozoïdes avec le glycérol avant congélation, qui différencie les techniques $B$ et $C$, n'a pas affecté, d'une façon significative dans notre expérience, le taux de fertilité obtenu après insémination artificielle.

in moyenne, 6o,3 p. Ioo des truies sont gestantes avec 8,3 embryons.

\section{INTRODUCTION}

Différentes techniques ont été proposées pour la congélation du sperme de verrat (GRAHAM et al., I97 I $b$; RICHTER et LIEDICKE, I972 ; EINARSSON et al., I973, I974; Paguignon et al., I974; PURSel et Johnson, I974; Salamon et Visser, 
I974). Elles varient entre elles par le temps de refroidissement de la semence, I,5 à 6 heures pour abaisser la température de $30^{\circ} \mathrm{C}$ à $5^{\circ} \mathrm{C}$, et par la température de la semence lors de $1^{\prime}$ addition du glycérol, $30^{\circ} \mathrm{C}, I^{\circ} \mathrm{C}$ ou $5^{\circ} \mathrm{C}$. Une analyse factorielle comparant ces différents paramètres a été réalisée (PAQUignon et al., I974); elle a contribué à déterminer la méthode qui permet d'obtenir le meilleur taux de spermatozoïdes réanimés après dégel et 3 heures d'incubation. L'objet du travail rapporté dans cette note était de tester le pouvoir fécondant des spermatozoïdes obtenus par les meilleures des combinaisons techniques testées in vitro.

\section{MATÉRIEL ETT MÉTHODES}

Après collecte manuelle et filtration sur de la gaze, l'éjaculat entier est partagé en trois fractions $(\mathrm{A}, \mathrm{B}, \mathrm{C})$ et centrifugé à $30^{\circ} \mathrm{C}$ pendant $\mathrm{I} 5 \mathrm{mn}$ à $800 \mathrm{~g}$.

Le culot de chaque échantillon est dilué une première fois ( $\mathrm{I} / 2 \mathrm{en}$ volume) avec un dilueur jaune d'œuf glucose (Polge et al., 1970) non glycérolé, puis la semence est soumise aux traitements suivants (fig. I).

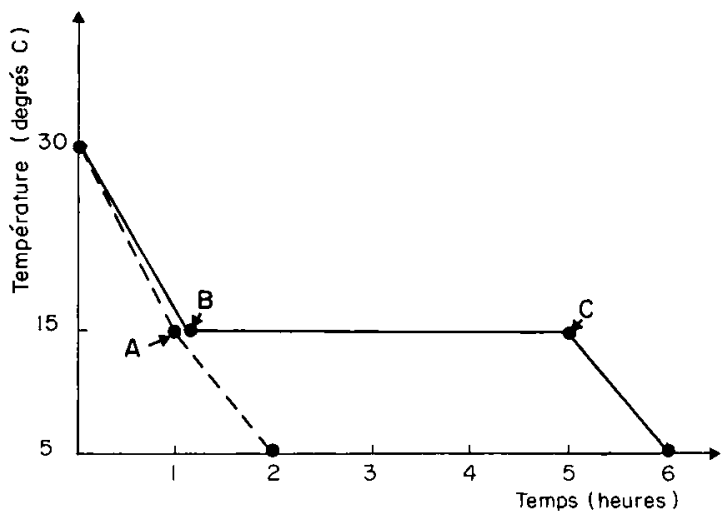

FIG. I. - Vitesse de refroidissement et moment de glycérolisation selon les différents traitements

I. - Vitesse de refroidissement

A : Abaissement de la température de $30^{\circ} \mathrm{C}$ à $15^{\circ} \mathrm{C}$ en $\mathrm{I}$ heure.

$\mathrm{B}$ et $\mathrm{C}$ : Abaissement de la température de $30^{\circ} \mathrm{C}$ à $15^{\circ} \mathrm{C}$ en $\mathrm{I}$ heure, maintien à $15^{\circ} \mathrm{C}$ pendant 4 heures.

Pour les trois traitements, abaissement de $15^{\circ} \mathrm{C}$ à $5^{\circ} \mathrm{C}$ en $\mathrm{I}$ heure.

\section{2. - Dilution glycérolée.}

Le même dilueur, mais additionné cette fois de glycérol aux taux de 6 p. Ioo en vue d'obtenir un taux final de 2,4 p. roo dans le sperme dilué, est ajouté à $15^{\circ} \mathrm{C}$ en quantité égale à celle utilisée pour la première dilution.

Pour A et $\mathrm{B}$ glycérolisation après $\mathrm{I}$ heure de refroidissement, dès que la température du sperme prédilué atteint ${ }^{\circ} 5^{\circ} \mathrm{C}$.

Pour C, glycérolisation après 5 heures de refroidissement, soit 4 heures après que le sperme prédilué ait atteint $15^{\circ} \mathrm{C}$.

A $5^{\circ} \mathrm{C}$, la semence est congelée en pastilles de o, I $\mathrm{ml}$ sur de la glace carbonique, puis trans- 
ferée dans l'azote liquide. Les doses ainsi obtenues sont décongelées dans le dilueur INRAITP à $50^{\circ} \mathrm{C}$ (I volume de sperme congelé pour 5 volumes de dilueur) (PAQuignon et du Mesnil du Buisson, I973). Les inséminations sont réalisées par pression à l'aide de la sonde de Melrose, Io à $15 \mathrm{mn}$ après décongélation en injectant $40 \mathrm{ml}$ de dilueur INRA-ITP avant et après la semence décongelée comprenant $3 \grave{a ̀ ~}_{4} \cdot{ }_{1}$ I $^{9}$ spermatozoïdes mobiles.

Nous avons utilisé des truies nullipares Large-White $\times$ Landrace. Le contrôle de l'uestrus est effectué deux fois par jour. Les truies sont inséminées 34 heures après le début présumé des chaleurs.

Une laparatomie pratiquée sur les truies non revenues en chaleur 30 à $4^{\circ}$ jours après l'insémination permet de compter par palper de l'utérus le nombre d'embryons présents. Cinq truies non contrôlées par laparoscopie sont allées jusqu'à la mise bas.

Vingt-deux éjaculats ont été traités pour l'insémination de 63 truies.

\section{RÉSUL,TATS}

Les résultats de fertilité et prolificité sont présentés dans le tableau I. Dans la limite de I à 5 heures, la durée de contact avant congélation entre les spermatozoïdes et le glycérol n'a pas d'influence significative sur le taux de gestation et le taux d'oufs fécondés (lots B et $\mathrm{C}$, tab1. I). Le maintien de la semence diluée 4 heures à $\mathrm{I}^{\circ} \mathrm{C}$ améliore significativement le taux de gestation $(\mathrm{P} \leqslant 0,0 \mathrm{I}$ lots $\mathrm{B}+\mathrm{C}$ contre A, tabl. I) sans modifier le taux d'cufs fécondés chez les truies pleines.

\section{TABLEAU I}

Fertilité et prolificité de truies inséminées une seule fois avec $3 \grave{a} 4 \cdot 10^{9}$ de spermatozoides vivants après décongélation

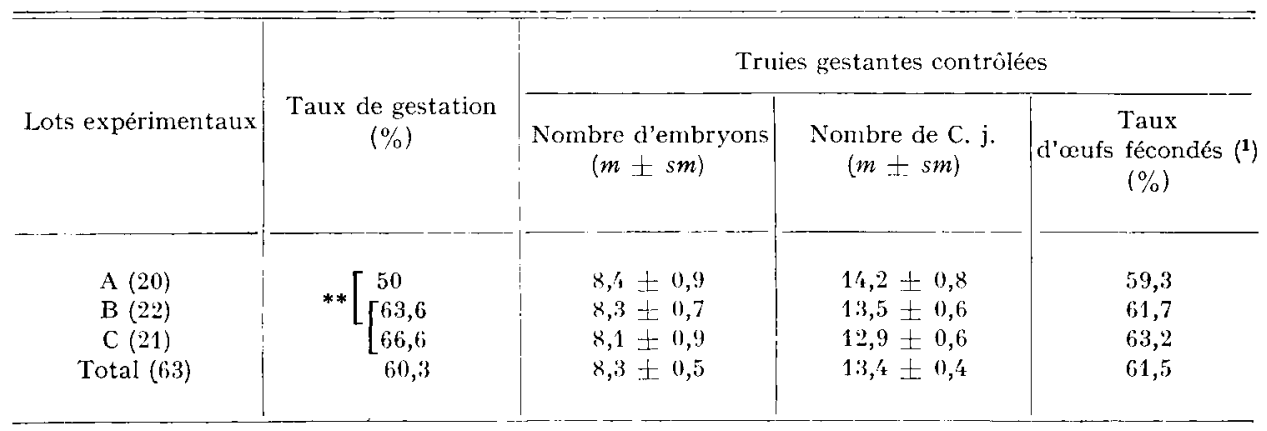

( ) Nombre d'animaux.

(1) Taux d'œufs fécondés : pourcentage d'embryons présents entre 30 et 40 jours de gestation par rapport au nombre des corps jaunes.

** : Difference hautement significative $(P \leqslant 0,(11)$.

Globalement, 6o,3 p. Ioo des truies sont gestantes. Le nombre de porcelets par portée est équivalent chez les truies contrôlées en cours de gestation $(8,3)$, et celles qui ont mis bas $(8,4)$. 


\section{DISCUSSION}

Les traitements où la semence diluée est maintenue à $15^{\circ} \mathrm{C}$ pendant 4 heures avant d'être congelée (lots $\mathrm{B}$ et $\mathrm{C}$ ) permettent d'obtenir un taux de gestation supérieur à celui obtenu avec la semence refroidie sans arrêt entre $30^{\circ} \mathrm{C}$ et $5^{\circ} \mathrm{C}$. Ce résultat est en accord avec les observations in vitro de la qualité du sperme selon le mode de préparation de la semence où le taux de réanimation et de survie du sperme maintenu 4 heures à $I^{\circ} \mathrm{C}$ était significativement supérieur à celui du sperme refroidi directement de $3^{\circ}$ à $5^{\circ} \mathrm{C}$ (PAQUiGNON et al., I974).

Ces résultats sont à rapprocher du fait que les techniques de congélation donnant de bons résultats de fertilité après insémination par voie cervicale emploient toute une durée de refroidissement avant congélation supérieure à 4 heures (RICHTER et Lifdicke, I972; Einarsson et al., 1973, 1974; PURsei. et Johnson, 1974).

Avec une vitesse de refroidissement plus rapide ( 2 h 30 à I h 30$)$ POLGE et al. (1970) n'obtiennent de truies gestantes qu'après insémination par voie chirurgicale et SALAMON et VISSER (I974) n'ont eu recours qu'à des inséminations chirurgicales dans les trompes. Les spermatozoïdes ainsi obtenus seraient dans l'incapacité de passer la jonction utérotubaire qui joue le rôle de filtre des spermatozoïdes selon leur qualité (HunTER, I973). Les conditions de refroidissement de la semence au cours de sa préparation doivent donc jouer un rôle important sur la qualité des spermatozoïdes après dégel. Un travail récent de microscopie électronique (MERGounis, I974) portant sur la comparaison de notre technique de congélation (méthode $c$ ) et de celle de Polge et al. (1970) vient en effet de montrer que la première respecte l'intégrité morphologique des spermatozoïdes significativement mieux que la seconde, elle permet aussi d'obtenir un meilleur taux de réviviscence au dégel (MERGounis, I974).

Le sperme congelé selon notre technique A pernet néanmoins d'obtenir $50 \mathrm{p}$. Ioo de truies gestantes après insémination par voie cervicale alors que PoLGE et al. (I970) employant une vitesse de refroidissement similaire et le même mode d'insémination n'obtiennent aucune gestation. Cette différence peut provenir de l'emploi, pour notre cas, d'une décongélation plus rapide dans un dilueur adapté. En effet, la vitesse et le dilueur de décongélation ont une influence significative sur la qualité des spermatozoïdes après dégel (Pursei, et JohnSon, I974).

Un autre test permettant de juger la qualité des spermatozoïdes est la mesure de la décharge de la transaminase glutamique oxaloacétique (GOT) de la cellule dans le milieu extérieur. Cette décharge est accrue quand la durée de contact entre le glycérol et les spermatozoïdes augmente (BowER ct al., I973). La technique B qui entraîne un contact des spermatozoïdes avec le glycérol de 4 heures de plus que la technique $\mathrm{C}$ n'affecte cependant pas le taux de fertilité obtenu après insémination avec du sperme congelé selon ces méthodes $(63,6 \mathrm{p}$. Ioo contre $66,6 \mathrm{p}$. Ioo respectivement). Si le taux moyen de fertilité que nous obtenons est encourageant, la prolificité est cependant inférieure d'un porcelet environ à celle couramment obtenue après insémination avec du sperme frais chez les truies nullipares. Il convient donc de poursuivre les travaux qui permettront d'améliorer encore ces résultats. 


\title{
CONCLUSION
}

I a technique de congélation de la semence de verrat incluant le maintien de la semence à $15^{\circ} \mathrm{C}$ pendant 4 heures est celle qui donne les meilleurs résultats de fertilité après insémination. Ces résultats sont convenables puisque dans le meilleur cas 66,6 p. Ioo des truies sont gestantes; cependant ceux-ci sont obtenus avec des inséminations pratiquées à un moment bien déterminé après le début de l'œstrus. La précision de la détection de l'œstrus semble dans le cas d'utilisation de sperme congelé un élément plus important encore que lorsque les inséminations sont réalisées avec du sperme frais.

Requ pour publication en mars 1975.

\section{REMERCIEMENTS}

Ce travail a été réalisé grâce à un financement du li. O. R. M. A. versé au titre d'une convention passée entre cet organisme, l'I. T. P. et l'L. N. R. A. dans le cadre du programme de Rationalisation de la Production Porcine.

\section{SUMMARY}

\author{
FERTIIITY AND PROI,IFICACY \\ OF SOWS INSEMINATED WITH FROZEN BOAR SEMEN
}

Three techniques for preparation of semen before freezing were studied in order to examine the fertilizing capacity of spermatozoa. Whole ejaculates of 9 boars were divided into 3 fractions $(\mathrm{A}, \mathrm{B}, \mathrm{C})$ and centrifuged at $800 \mathrm{~g}$ for $15 \mathrm{mn}$ at $30^{\circ} \mathrm{C}$. The precipitate was diluted with an unglycerolated glucose-egg-yolk diluent followed by cooling of the semen at two rates : group A : $30^{\circ} \mathrm{C}$ to $15^{\circ} \mathrm{C}$ within $\mathrm{I}$ hour, $15^{\circ} \mathrm{C}$ to $5^{\circ} \mathrm{C}$ within $\mathrm{I}$ hour. Groups $\mathrm{B}$ and $\mathrm{C}: 30^{\circ} \mathrm{C}$ to $15^{\circ} \mathrm{C}$ within $\mathrm{I}$ hour, holding at $\mathrm{I}^{\circ} \mathrm{C}$ for 4 hours and cooling to $5^{\circ} \mathrm{C}$ within $\mathrm{I}$ hour. The semen was rediluted with the same diluent, but with glycerol at $\mathrm{I}_{5}^{\circ} \mathrm{C}$ after $\mathrm{I}$ hour of cooling for groups $\mathrm{A}$ and $\mathrm{B}$ and 5 hours of cooling for group $C$. The semen was frozen in pellets of $O$. I ml. Insemination doses were thawed in the INRA-ITP diluent at $50^{\circ} \mathrm{C}$ ( $\mathrm{I}: 5$ ). 63 nulliparous sows were inseminated with 3 to $4 \cdot 10^{9}$ motile spermatozoa 34 hours after the presumed onset of heat.

Holding the semen for 4 hours at $15^{\circ} \mathrm{C}$ significantly improved $(\mathbf{P} \leqslant 0.05)$ the conception rate $(63.6 \mathrm{p}$. Ioo) and $66.0 \mathrm{p}$. roo for groups $B$ and $C$ versus 50 p. roo for group A).

The 4 hours-increase in the duration of contact of spermatozoa with glycerol before freezing (differentiating technique B from () did not, in our experimental conditions, significantly affect the fertility rate obtained alter artificial insemination.

On an average, $60.3 \mathrm{p}$. Ioo of the sows were pregnant with 8.3 embryos.

\section{RÉFÉRENCES BIBLIOGRAPHIQUES}

Bower R. E., Crabo B. G., Pace M. M., Graham E. li., I973. Effects of dilution and glycerol on the release of Glutanic oxaloacetic transaminase (GOT) from boar spermatozoa. J. Anim. Sci., 36, 319-324.

Einarsson S., Swensson T., Viring S., 1973. A field trial on the fertility of deep-frozen boar spermatozoa. Nord. Vet. Med., 25, 372-376. 
Linarsson S., Holtman H., Soosalu O., Swensson T., Viring S., I974. Studies on the fertility and survival of deep-frozen boar spermatozoa thawed in four different diluents. Zuchthyg., 9, 40-45.

Graham E. F, Rajamannan a. H. J., Scheil. H. K., L., Maki-Lavria M., Bower R. E., ig7i $b$. Fertility studies with frozen boar spermatozoa. A. I. Dig., 19, 6-8.

Hunter R. H. F., 1973. Transport, immigration and survival of spermatozoa in the female genital tract : species with intra-uterine deposition of semen. I. N. S. E. R. M., 26, 309-342.

MERgounis D. J., I974. Modifications ultrastructurales, cytochimiques et enzymatiques des spermatozoïdes de verrat après congélation. Thèse de Docteur Ingénieur. Université Pierre et Marie Curie (Paris VI), $37 \mathrm{p}$.

Paguignon M., du Mesnil du Buisson F., 1973. Fertilité et piolificité de truies inséminées avec du sperme congelé. Journées Rech. Porcine en France, 49-57, Paris, I. T. P. éd.

Paguignon M., Mergounis D., Courot M., du Mesnil du Buisson F., i974. Technologie de la congélation de la semence de verrat: étude in vitro. Journées Rech. Porcine en France, $71-76$, Paris, I. T. P. éd.

Polge C., Salamon S., Wilmut I., 1970. Fertilizing capacity of frozen boar semen following surgical insemination. Vet. Rec., 87, 424-428,

Pursel V. G., Johnson L. A., I974. Frozen boar spermatozoa : Fertility with concentrated semen and a new thawing procedure. $J$. Anim. Sci., 39, 222 (Abstr.).

Pursel V. G., Johnson L. A., r974. Frozen boar spermatozoa : Methods of thawing. J. Anim. Sci., 39, 222 (Abstr.).

Richter L., Liedicke A., 1972. Method of deep-freezing boar semen. Proc. VIIth Int. Congr. Anim. Reprod. München, II, 1617-1621.

Salamon S., Visser D., 1974. Fertility after surgical insemination with frozen boar semen. Aust. J. Biol. Sici., 27, 499-504. 